\title{
The Usefulness of Intensivist-Performed Bedside Drainage of Pleural Effusion via Ultrasound-Guided Pigtail Catheter
}

Joo-Won Min, M.D., Joon Young Ohm, M.D. , , Byung Seok Shin, M.D. , Jun Wan Lee, M.D. ${ }^{\dagger}$, Sang-Il Park, M.D. ${ }^{\ddagger}$, Seok Hwa Yoon, M.D. ${ }^{\ddagger}$, Yong Sup Shin, M.D. ${ }^{\ddagger}$, Dong-Il Park, M.D. ${ }^{\S}$, Chaeuk Chung, M.D. ${ }^{\S}$, and Jae Young Moon, M.D. ${ }^{\S}$

Division of Pulmonary and Critical Care Medicine, Department of Internal Medicine, Myongji Hospital, Goyang; "Department of Radiology, Chungnam National University College of Medicine, Daejeon; ${ }^{\dagger}$ Emergency Intensive Units, Daejeon Regional Emergency Center, Chungnam National University College of Medicine, Daejeon; ${ }^{\ddagger}$ Department of Anesthesiology and Pain Medicine, Chungnam National University College of Medicine, Daejeon; ${ }^{\S}$ Division of Pulmonary and Critical Care Medicine, Department of Internal Medicine, Chungnam National University College of Medicine, Daejeon, Korea

Background: There has been little data reporting the usefulness of intensivist-performed bedside drainage of pleural effusion via ultrasound (US)-guided pigtail catheter. The objective of this study is to clarify the usefulness and safety of these methods in comparison with radiologist-performed procedures.

Methods: Data of patients with pleural effusion treated with US-guided pigtail catheter drainage were analyzed. All procedures were performed from September 2012 to September. 2013 by a well-trained intensivist or radiologist.

Results: Pleural effusion was drained in 25 patients in 33 sessions. A radiologist performed 21 sessions, and an intensivist performed 12 sessions. Procedures during mechanical ventilation were performed in $15(71.4 \%)$ patients by a radiologist and in $10(83.3 \%)$ by an intensivist $(\mathrm{p}=0.678)$. The success rate was not significantly different in radiologist- and intensivist-performed procedures, $95.2 \%$ $(20 / 21)$ and $83.3 \%(10 / 12)$, respectively $(p=0.538)$. The average duration for procedures (including in-hospital transfer) was longer in radiologist-performed cases $(\mathrm{p}=0.001)$. Although the results are limited because of the small population size, aggravation of oxygenation, $\mathrm{CO}_{2}$ retention, and decrease of mean arterial blood pressure were not statistically different in the groups. Pigtail-associated complications including hemothorax, pneumothorax, hepatic perforation, empyema, kink in the catheter, and subcutaneous hematoma were not found.

Conclusions: Intensivist-performed bedside drainage of pleural effusion via ultrasound (US)-guided pigtail catheter is useful and safe and may be recommended in some patients in an intensive care unit.

Key Words: drainage; pleural effusion; ultrasonography.

\section{Introduction}

Pleural effusion (PE) is caused by many different diseases and

Received on December 23, 2013 Revised on May 6, 2014

Accepted on May 14, 2014

Correspondence to: Jae Young Moon, Division of Pulmonary and Critical Care Medicine, Department of Internal Medicine, Chungnam National University College of Medicine, 282 Munhwa-ro, Jung-gu, Daejeon 301-721, Korea

Tel: +82-42-280-7870, Fax: +82-42-280-7877

E-mail:jymoon@cnuh.co.kr

* No potential conflict of interest relevant to this article was reported. classified as exudate or transudate, depending on the cause of the effusion to be formed. Malignancy, pneumonia, tuberculosis are common cause of exudative PE while heart failure is largely responsible for transudative PE.[1] PE commonly occurs in critically ill patients although the incidence varies in the range of $8 \%$ to $60 \%,[2-4]$ and ultrasound (US) of the chest is commonly used to diagnose PE.[5]

Percutaneous drainage of PE via pigtail catheters has been widely used to improve oxygenation and facilitate mechanical ventilation[6-8] although the evidence was controversial among many researchers. $[9,10]$ In 2009, a Taiwanese study of 133 critically ill patients reported a $58 \%$ of success rate in the drainage 
procedure of PE.[11] However, no Korean studies have ever been done about the US-guided drainage by intensivists in ICU because few ICUs were equipped with US units in Korea. Because the transfer of patients for drainage procedures can cause undesirable problems of patients' safety,[12] especially in patients with mechanical ventilation or continuous renal replacement therapy, it would be helpful to drain PE in ICU by intensivistperformed procedures.

This study aimed to investigate the effectiveness and safety of the US-guided effusion drainage via pigtail-catheter insertion by an intensivist, and compare clinical outcomes between intensivistand interventional radiologist-performed procedures.

\section{Materials and Methods}

\section{1) Data collection}

Retrospective analysis was performed using the medical records of ICU patients who underwent US-guided pigtail catheter drainage of $\mathrm{PE}$ at the Chungnam National University Hospital from September 2012 to September 2013. The drainage procedures were performed by either an ICU intensivist or an interventional radiologist. This study was approved by institutional review board and research ethics committee (IRB No. CNUH 2013-12-027).

\section{2) Catheterization process}

Procedures by a radiologist were performed in an interventional radiology suite like other radiological procedures. In mechanically ventilated patients, Bag-mask ventilation was used for temporary ventilation during the catheter insertion. All catheter insertions by an intensivist were conducted at the bedside of ICU. During the procedures of mechanically ventilated patients, each patient was placed in semi flower position with or without an opposite side decubitus elevated by 15 degrees, on the physiologic positive end-expiratory pressure (PEEP) level.

Full sterile barrier precautions were followed in all procedures. An $8.5 \mathrm{~F}$ pigtail catheter (Cook, Bloomington, IN, USA) was inserted using the modified Seldinger technique for each patient.

US-guided catheter insertion was performed by a radiologist as follows: 1) a suspected PE by chest X-ray or CT scan was examined using ultrasound for further analysis, 2) an insertion site was marked and sterilized with betadine, 3 ) the chest wall was pierced with a 16- or 18-gauge angiocath needle or a 22 -gauge Chia needle with the guidance of ultrasound, 4) a 0.035 inch induction guide wire was then advanced to the appropriate pleural space us- ing fluoroscopy, 5) after the insertion site was enlarged with an $8.5 \mathrm{~F}$ dilator, an $8.5 \mathrm{~F}$ catheter was inserted into the pleural space, and 6) the catheter was fixed to the skin. Catheter insertions by an intensivist were performed using the same techniques in the ICU setting except the step for the use of fluoroscopy.

Catheterization was performed in cases with classic indications for catheter insertion such as empyema and malignant PE. Even in patients without typical indications, effusion was drained by the pigtail catheter to facilitate mechanical ventilation if necessary. The results of procedures were classified into 'success' if the volume of PE was reduced on Chest X-rays after procedures or 'failure' if the volume was not reduced. If the large amount of remaining fluid did not drain out easily even when the procedure was successful, the position of catheter tip was examined by ultrasound and adjusted. However, if no fluid still drained out, the case was regarded as catheter malfunction. Complications of catheter insertions such as hemothorax, pneumothorax, liver perforation, empyema, damage to internal organs by a kink of catheter, and severe subcutaneous hematoma were checked after each procedure.

\section{3) Data analysis}

We compared the outcomes of US-guided pigtail catheter insertions performed by an intensivist at the bedside of ICU and that by a radiologist for patients transferred to the interventional radiology suite. Outcomes of procedures were evaluated according to in-ICU mortality, procedural success rate, the amount of PE drained until one day and three days after catheter insertion, and the duration of procedures. Also clinical parameters were analyzed to identify procedure-related complications, intra-hospital transport complication, especially in patients undergoing mechanical ventilation and continuous renal replacement therapy. These complications included deteriorated oxygenation, accumulation of carbon dioxide, and low blood pressure caused by the accidental cessation of vasopressor infusion.

The independent sample t-test was used for comparisons of continuous variables (the Mann-Whitney test for nonparametric variables), and the Chi-square test or Fisher's exact test was used for categorical data. Statistical analysis was performed using SPSS 18.0 (SPSS Inc., Chicago, IL, USA).

\section{Results}

\section{1) Clinical parameters associated with baseline characteristics}


A total of 25 ICU patients underwent US-guided pigtail catheter drainage of PE, and the number of procedures was 33. Of those, 21 sessions were performed by a radiologist and 12 were by an intensivist. Of total patients, eight underwent the drainage procedure twice, and seven of them underwentd the procedures at least 4-day interval. Because the procedures were performed in variable clinical settings, this study was analyzed based on the number of procedure. There were no differences between patients in both groups with respect to main diagnosis and disease distribution (Table 1) except for a greater rate of respiratory failure

Table 1. Underlying disease of analyzed 25 patients

\begin{tabular}{lccr}
\hline \hline & \multicolumn{2}{c}{ Intervention by } & \\
\cline { 2 - 3 } & $\begin{array}{c}\text { Radiologist } \\
(\mathrm{n}=15)\end{array}$ & $\begin{array}{c}\text { Intensivist } \\
(\mathrm{n}=10)\end{array}$ & \\
\hline Diabetes mellitus & $6(40.0)$ & $2(20.0)$ & 0.402 \\
Cardiovascular diseases & $8(53.3)$ & $5(50.0)$ & $>0.999$ \\
Respiratory failure & $6(40.0)$ & $8(80.0)$ & 0.099 \\
Septic shock & $4(26.7)$ & $0(0.0)$ & 0.125 \\
Neurologic diseases & $1(6.7)$ & $2(20.0)$ & 0.543 \\
Malignant diseases & $4(26.7)$ & $3(30.0)$ & $>0.999$ \\
Renal diseases & $5(33.3)$ & $5(50.0)$ & 0.442 \\
Liver cirrhosis & $2(13.3)$ & 0 & 0.500 \\
After intraabdominal surgery & $5(33.3)$ & $2(20.0)$ & 0.659 \\
\hline
\end{tabular}

Values are presented as number (\%).

Table 2. Clinical parameters associated with 33 procedures in 25 patients in the intensivist-performed group, which was not statistically significant $(p=0.099)$.

Clinical parameters associated with procedures were presented in Table 2. A larger number of patients had effusion in right side, and exudate was dominant. In 18 cases, because the catheter drainage was performed to facilitate weaning from mechanical ventilator or improve symptom and sign associated with critical care, the characteristics of effusion was not analyzed in most patients (transudate in some patients).

The cases of mechanically ventilated patients constituted $71.4 \%(15 / 21)$ of the radiologist-performed group and $83.3 \%$ $(10 / 12)$ of the intensivist-performed group, showing no statistical difference. The patients undergoing continuous renal replacement therapy comprised $25.0 \%$ (3/12) of the intensivist-performed group, but no patient was found in the radiologist-performed group $(p=0.040)$. There were no differences between both groups in severity of diseases (APACHE-II score), the proportion of patients with large amount of effusion $(\geq 50 \%$ of lung field in simple $\mathrm{X}$-ray), and the proportion of patients using vasopressors. Although the parameters associated with difficulties of procedures such as coagulation disorder (e.g., abnormalities of platelet count, international normalized ratio (INR), and activated partial

\begin{tabular}{|c|c|c|c|}
\hline & \multicolumn{2}{|c|}{ Intervention by } & \multirow{2}{*}{$\mathrm{p}$ value } \\
\hline & Radiologist $(\mathrm{n}=21)$ & Intensivist $(\mathrm{n}=12)$ & \\
\hline Age, yr (range) & $78(46-90)$ & $71.5(43-91)$ & 0.368 \\
\hline Site & & & $>0.999$ \\
\hline Right & $15(71.4)$ & $9(75.0)$ & \\
\hline Left & $6(28.6)$ & $3(25.0)$ & \\
\hline \multicolumn{4}{|l|}{ Fluid analysis } \\
\hline Transudate & $4(19.0)$ & $1(8.3)$ & \\
\hline Exudate other than malignant effusion & $6(28.6)$ & $7(58.3)$ & \\
\hline Malignant effusion & $2(9.5)$ & 0 & \\
\hline Not analyzed & $9(42.9)$ & $4(33.3)$ & \\
\hline \multicolumn{4}{|l|}{ Life saving devices } \\
\hline Mechanical ventilation & $15(71.4)$ & $10(83.3)$ & 0.678 \\
\hline $\mathrm{PaO}_{2} / \mathrm{FiO}_{2}$ before procedure (mean $\pm \mathrm{SD}$ ) & $214.31 \pm 86.09(\mathrm{n}=15)$ & $171.46 \pm 91.79(\mathrm{n}=9)$ & 0.262 \\
\hline Renal replacement therapy & 0 & $3(25.0)$ & 0.040 \\
\hline APACHE II score (mean \pm SD) & $19.48 \pm 7.15$ & $20.83 \pm 8.62$ & 0.652 \\
\hline Large amount of effusion ( $\geq 50 \%$ of lung field $\left.{ }^{*}\right)$ & $15(71.4)$ & $9(75.0)$ & $>0.999$ \\
\hline Use of inotropic agents/vasopressor & $3(14.3)$ & $2(16.7)$ & $>0.999$ \\
\hline \multicolumn{4}{|l|}{ Coagulation abnormality } \\
\hline Platelet count $<100,000 / \mathrm{mm}^{3}$ & $7(33.3)$ & $4(33.3)$ & $>0.999$ \\
\hline INR $>1.5$ & $3(14.3)$ & $2(20.0 ; \mathrm{n}=10)$ & $>0.999$ \\
\hline $\mathrm{aPTT}>60 \mathrm{sec}$ & $3(15.0 ; n=20)$ & $2(20.0 ; \mathrm{n}=10)$ & $>0.999$ \\
\hline Drugs affecting ineffective bleeding control & & & 0.030 \\
\hline Antiplatelet agents & $0(0.0)$ & $3(25.0)$ & \\
\hline Anticoagulation & $2(9.5)$ & $2(16.7)$ & \\
\hline Body mass index $\geq 25$ & $2(10.5)$ & $2(18.2)$ & 0.611 \\
\hline
\end{tabular}

Values are presented as number (\%) unless otherwise indicated. *Amount of effusion was evaluated with simple X-ray. APACHE: acute physiology and chronic health evaluation; INR: international normalized ratio; aPTT: activated partial thromboplastin time. 
Table 3. Outcomes of intervention

\begin{tabular}{|c|c|c|c|}
\hline & \multicolumn{2}{|c|}{ Intervention by } & \multirow{2}{*}{$\mathrm{p}$ value } \\
\hline & Radiologist $(\mathrm{n}=21)$ & Intensivist $(\mathrm{n}=12)$ & \\
\hline In-ICU mortality & $8(38.1)$ & $7(58.3)$ & 0.261 \\
\hline Successful intervention & $20(95.2)$ & $10(83.3)$ & 0.538 \\
\hline \multicolumn{4}{|l|}{ Amount of drainage, $\mathrm{ml}$} \\
\hline-1 st day & $840.45 \pm 330.01$ & $852.92 \pm 279.85$ & 0.969 \\
\hline$-3 r d$ day & $1424.55 \pm 722.23$ & $1666.50 \pm 678.30$ & 0.333 \\
\hline Duration of procedure (including transfer), min & $44.75 \pm 14.65$ & $23.75 \pm 11.26$ & 0.001 \\
\hline Major complications" & 0 & 0 & $>0.999$ \\
\hline \multicolumn{4}{|l|}{ Physiologic changes after intervention } \\
\hline $\mathrm{PaO}_{2}$ (after) $-\mathrm{PaO}_{2}$ (before), $\mathrm{mmHg}$ & $3.38 \pm 47.64$ & $15.82 \pm 23.58$ & 0.424 \\
\hline Deterioration of oxygenation $^{\dagger}$ & $8(40.0 ; n=20)$ & $1(9.1 ; \mathrm{n}=11)$ & 0.106 \\
\hline $\mathrm{PaCO}_{2}$ (after)- $\mathrm{PaCO}_{2}$ (before), $\mathrm{mmHg}$ & $2.15 \pm 8.51$ & $0.00 \pm 7.40$ & 0.488 \\
\hline $\mathrm{CO}_{2}$ retention after intervention $\geq 10 \mathrm{mmHg}$ & $3(15.0 ; n=20)$ & $2(18.2 ; \mathrm{n}=11)$ & $>0.999$ \\
\hline MABP (after)-MABP (before), $\mathrm{mmHg}$ & $-9.20 \pm 15.59$ & $-11.83 \pm 19.39$ & 0.676 \\
\hline MABP $<70 \mathrm{mmHg}$ (after intervention) & $3(15.0 ; n=20)$ & $1(8.3)$ & $>0.999$ \\
\hline
\end{tabular}

Values are presented as mean $\pm \mathrm{SD}$ or number (\%). " hemothorax, pneumothorax, hepatic perforation, empyema, a kink in the catheter, and subcutaneous hematoma. ${ }^{\dagger} \mathrm{PaO}_{2} / \mathrm{FiO}_{2}$ (after procedure) - $\mathrm{PaO}_{2} / \mathrm{FiO}_{2}$ (before procedure) < 0 . ICU: intensive care unit; MABP: mean arterial blood pressure.

thromboplastin time) and obesity (body mass index, BMI) were not different between two groups, the number of cases with antiplatelet or anticoagulation therapy was greater in intensivistperformed group $(2 / 21 \mathrm{vs.} 5 / 12, \mathrm{p}=0.030)$.

\section{2) The results of US-guided pigtail catheter drainage}

US-guided pigtail catheter drainage had success rate of $95.2 \%$ $(20 / 21)$ and $83.3 \%(10 / 12)$, respectively, in the radiologist- and intensivist-performed groups, and in-ICU mortality was $38.1 \%$ $(8 / 21)$ in the radiologist-performed group and $58.3 \%(7 / 12)$ in the intensivist-performed group (Table 3). Both success rate and in-ICU mortality showed no significant differences between two groups. Three cases had procedural failures, and two of them were conducted by an intensivist. Procedures were not successful in short and overweight patients with BMI of $22.9 \mathrm{~kg} / \mathrm{m}^{2}$ and 23.0 $\mathrm{kg} / \mathrm{m}^{2}$, respectively, because their intercostal space was too narrow for the insertion of catheter. In these patients, simple pleurocentesis was carried out and the volume of manually drained effusion was $645 \mathrm{ml}$ and $415 \mathrm{ml}$, respectively. One procedural failure was performed by a radiologist, and no PE was drained in this case. The radiologist pursued no alternative but used a wait-andsee approach in this case because the volume of PE was not large.

The volume of drained effusion until one day or three days after the procedures was not significantly different in both groups. However, the radiologist-performed procedures took a significantly longer time because the time taken for intra-hospital transport was included $(\mathrm{p}=0.001)$. We also compared changes in mean arterial blood pressure (MABP), partial pressure of arterial oxygen $\left(\mathrm{PaO}_{2}\right)$ and carbon dioxide $\left(\mathrm{PaCO}_{2}\right)$. There were no changes of $\mathrm{PaO}_{2}$ in both groups. Deterioration of oxygenation (increase of $\mathrm{PaO}_{2} / \mathrm{FiO}_{2}$ ratio) after procedures was found in $40.0 \%(8 / 20)$ of the radiologist-performed group and in $9.1 \%$ $(1 / 11)$ of the intensivist-performed group. But the difference was not statistically significant $(\mathrm{p}=0.106)$. Changes of $\mathrm{PaCO}_{2}$ after the procedures and the proportion of patients showing $\geq 10$ $\mathrm{mmHg}$ rise in $\mathrm{PaCO}_{2}$ were not different between two groups. And the significant differences were also not found in changes of MABP after the procedures and the proportion of patients showing $<70 \mathrm{mmHg}$ decrease in MABP.

In both groups, hemothorax, pneumothorax, liver perforation, empyema, damage to internal organs by a kink of catheter, and severe subcutaneous hematoma were not found.

\section{Discussion}

This study ascertains the issues involved in radiologist-performed procedures for ICU patients transferred to the radiology suite. In most of Korean general hospitals (even in tertiary teaching centers), it is hard to assign radiologists temporarily to the ICU for interventional procedures, and it is uncertain whether they can provide the required procedure on a timely manner like intensivists. Transport of critically ill patients out of the ICU is also discouraging because their diminished physiologic reserve increases the risk of critical complications threatening patients' safety, thus outof-ICU procedures and treatment should be decided according to the patients clinical status. For this reason, we investigated the effectiveness and safety of US-guided catheter drainage performed by an intensivist on a timely manner instead of transport to intervention room. The outcome of procedures by an intensivist was 
then compared with those by a radiologist, and no statistical differences were observed in the comparisons.

In addition, although undesirable conditions such as continuous renal replacement therapy $(p=0.040)$, drugs affecting ineffective bleeding control $(p=0.030)$ were observed in the intensivist-performed group, no major complications were found and the procedural success rate reached $83.3 \%$, which was similar to that of radiologist-performed procedures $(p=0.538)$. Despite the concerns about transport related complications outside of ICU, cases in the radiologist-performed group did not significantly develop any significant complications such as low blood pressure by the accidental cessation of vasopressor infusion, deteriorated oxygenation or accumulation of carbon dioxide as a result of ventilator disconnection during transfer to the radiology suite.

In the intensivist-performed group, the proportion of cases whose oxygenation levels $\left(\mathrm{PaO}_{2} / \mathrm{FiO}_{2}\right.$ ratio) deteriorated after procedures was seemed to be lower, compared with the radiologist-performed group ( $9.1 \%$ vs. $40.0 \%)$, and the patients whose MABP $<70 \mathrm{mmHg}$ were also seemed to be less $(8.3 \%$ vs. $15.0 \%)$. Moreover, an intensivist required far less procedure time than a radiologist $(23.75 \pm 11.26 \mathrm{~min}$ vs. $44.75 \pm 14.65 \mathrm{~min} ; \mathrm{p}=$ 0.001 ), increasing the safety of the procedures. Because two failed intensivist-performed procedures were attributable to the narrow intercostal space of patients, intensivists need to take extra caution to perform procedures in this type of patients.

As the safety of US-guided procedures is increasingly proven, [13] the attempts of PE drainage have been made in the ER and ICU settings without the help of radiologists. A study reported successful drainage in $72.9 \%$ of 276 patients with PE when the drainage procedure was performed by emergency physicians or ICU intensivists,[14] and another study claimed the success rate of intensivist-performed drainage was $58 \%$ in 133 patients.[11] Although their success rates were lower than that of this study, the simple comparison may be inappropriate given that the success rate is also affected by clinical factors such as the characteristics of PE and the inner diameter of catheter. For the fibrotic septation or organization of pleural fluid, which is not detected by chest $\mathrm{CT}$, increases the risk of procedural failure, these would be important factors influencing the success rate of procedures.

No major complications associated with catheterization were developed. Local infection was reported in several previous studies,[14] but it was not found in this study. Although there could be several unrecorded complications, there was no way to confirm them in a retrospective study.

This is the first Korean study to clarifying the effectiveness and safety of US-guided pigtail catheter drainage performed by an in- tensivist by comparing the outcomes of procedures with those of radiologist-performed procedures. This study found no statistical differences between the two groups in baseline conditions of each case and clinical outcomes after procedures. And, it was confirmed that intensivists might be in a noninferior position to carry out procedures safely without the need for patient transport. This study however has limitations, including its retrospective design and sample size, which is too small for statistical tests of significance. Further limitations include the difficulty in comparing malfunction-free period between the two groups because the timing of catheter removal varied among involved physicians. Further prospective studies are needed to address this issue. Because inexperience of the intensivist could be the cause of two procedural failures, further non-inferiority trials must be performed.

In conclusion, an intensivist-performed bedside drainage of pleural effusion via US-guided pigtail catheter is useful and safe, and can be considered in some patients at risk of deterioration to be transferred from the ICU.

\section{References}

1) Marel M, Zrustova M, Stasny B, Light RW: The incidence of pleural effusion in a well-defined region. Epidemiologic study in central Bohemia. Chest 1993; 104: 1486-9.

2) Mattison LE, Coppage L, Alderman DF, Herlong JO, Sahn SA: Pleural effusions in the medical ICU: prevalence, causes, and clinical implications. Chest 1997; 111: 1018-23.

3) Fartoukh M, Azoulay E, Galliot R, Le Gall JR, Baud F, Chevret S, et al: Clinically documented pleural effusions in medical ICU patients: how useful is routine thoracentesis? Chest 2002; 121: 178-84.

4) Azoulay E: Pleural effusions in the intensive care unit. Curr Opin Pulm Med 2003; 9: 291-7.

5) Beckh S, Bölcskei PL, Lessnau KD: Real-time chest ultrasonography: a comprehensive review for the pulmonologist. Chest 2002; 122: 1759-73.

6) Talmor M, Hydo L, Gershenwald JG, Barie PS: Beneficial effects of chest tube drainage of pleural effusion in acute respiratory failure refractory to positive end-expiratory pressure ventilation. Surgery 1998; 123: 137-43.

7) Gillespie DJ, Rehder K: Effect of positional change on ventilation-perfusion distribution in unilateral pleural effusion. Intensive Care Med 1989; 15: 266-8.

8) Tu CY, Hsu WH, Hsia TC, Chen HJ, Chiu KL, Hang LW, et al: The changing pathogens of complicated parapneumonic 
effusions or empyemas in a medical intensive care unit. Intensive Care Med 2006; 32: 570-6.

9) Lai YF, Chao TY, Wang YH, Lin AS: Pigtail drainage in the treatment of tuberculous pleural effusions: a randomised study. Thorax 2003; 58: 149-51.

10) Parker LA, Charnock GC, Delany DJ: Small bore catheter drainage and sclerotherapy for malignant pleural effusions. Cancer 1989; 64: 1218-21.

11) Liang SJ, Tu CY, Chen HJ, Chen CH, Chen W, Shih CM, et al: Application of ultrasound-guided pigtail catheter for drainage of pleural effusions in the ICU. Intensive Care Med
2009; 35: 350-4.

12) Fanara B, Manzon C, Barbot O, Desmettre T, Capellier G: Recommendations for the intra-hospital transport of critically ill patients. Crit Care 2010; 14: R87.

13) Mynarek G, Brabrand K, Jakobsen JA, Kolbenstvedt A: Complications following ultrasound-guided thoracocentesis. Acta Radiol 2004; 45: 519-22.

14) Liu YH, Lin YC, Liang SJ, Tu CY, Chen CH, Chen HJ, et al: Ultrasound-guided pigtail catheters for drainage of various pleural diseases. Am J Emerg Med 2010; 28: 915-21. 